\title{
Modeling Multibody Stage Separation Dynamics Using Constraint Force Equation Methodology
}

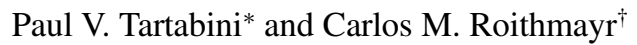 \\ NASA Langley Research Center, Hampton, Virginia 23681-2199 \\ Matthew D. Toniolo $\$$ and Christopher D. Karlgaard \\ Analytical Mechanics Associates, Inc., Hampton, Virginia 23681 \\ and \\ Bandu N. Pamadi $\underline{\S}$ \\ NASA Langley Research Center, Hampton, Virginia 23681-2199
}

DOI: $\underline{10.2514 / 1.51943}$

\begin{abstract}
This paper discusses the application of the constraint force equation methodology and its implementation for multibody separation problems using three specially designed test cases. The first test case involves two rigid bodies connected by a fixed joint, the second case involves two rigid bodies connected with a universal joint, and the third test case is that of Mach 7 separation of the $\mathrm{X}-43 \mathrm{~A}$ vehicle. For the first two cases, the solutions obtained using the constraint force equation method compare well with those obtained using industry- standard benchmark codes. For the $X-43 \mathrm{~A}$ case, the constraint force equation solutions show reasonable agreement with the flight-test data. Use of the constraint force equation method facilitates the analysis of stage separation in end-to-end simulations of launch vehicle trajectories.
\end{abstract}

\section{Nomenclature}

$\underline{A}, \underline{B}$

$\bar{A}, \bar{B}$

$a_{x}, a_{y}, a_{z}$

$\mathbf{F}_{A}^{(\mathrm{CON})}, \mathbf{F}_{B}^{(\mathrm{CON})}$

$\mathbf{F}_{A}^{(\mathrm{EXT})}, \mathbf{F}_{B}^{(\mathrm{EXT})}$

$F_{x}^{(\mathrm{CON})}, F_{y}^{(\mathrm{CON})}$,

$F_{x}^{(\mathrm{EXT})}, F_{y}^{(\mathrm{EXT})}$,

$F_{z}^{(\mathrm{EXT})}$

$\mathbf{I}_{A}, \mathbf{I}_{B}$

$m_{A}, m_{B}$

N

$\mathbf{r}_{A}, \mathbf{r}_{B}$

$\mathbf{T}_{A}^{(\mathrm{CON})}, \mathbf{T}_{B}^{(\mathrm{CON})}$

$\mathbf{T}_{A}^{(\mathrm{EXT})}, \mathbf{T}_{B}^{(\mathrm{EXT})}$

$T_{x}^{(\mathrm{CON})}, T_{y}^{(\mathrm{CON})}$, $T_{z}^{(\mathrm{CON})}$

$V_{x}, V_{y}, V_{z}$ body $B, \mathrm{lb}_{f}$ body $B, \mathrm{lb}_{f}$ frame, $l_{f}$ frame, $\mathrm{lb}_{f}$ and body $B$, slug $\cdot \mathrm{ft}^{2}$ body $B, \mathrm{ft} \cdot \mathrm{lb}_{f}$ frame, $\mathrm{ft} \cdot \mathrm{lb}_{f}$
$=$ rigid body $A$ and rigid body $B$

$=$ joint location in body $A$ and body $B$

$=$ components of sensed mass center acceleration in local body frame, $\mathrm{ft} / \mathrm{s}^{2}$

$=$ joint constraint force vector for body $A$ and

$=$ external force vector acting on body $A$ and

$=$ constraint force components in local body

$=$ external force components in local body

$=$ inertia tensor about mass center for body $A$

$=$ mass of body $A$ and body $B$, slug

$=$ number of equations and unknowns required to solve constraint force equation problem

$=$ inertial position vector of $A$ and $\bar{B}, \mathrm{ft}$

$=$ joint constraint torque vector for body $A$ and body $B, \mathrm{ft} \cdot \mathrm{lb}_{f}$

$=$ external torque vector acting on body $A$ and

$=$ constraint torque components in local body

$=$ inertial components of mass center velocity relative to inertial reference frame, $\mathrm{ft} / \mathrm{s}$
Presented as Paper 2008-7039 at the AIAA Modeling and Simulation Technologies Conference and Exhibit, Honolulu, HI, 18-21 August 2008; received 10 August 2010; revision received 5 April 2011; accepted for publication 20 April 2011. This material is declared a work of the U.S. Government and is not subject to copyright protection in the United States. Copies of this paper may be made for personal or internal use, on condition that the copier pay the $\$ 10.00$ per-copy fee to the Copyright Clearance Center, Inc., 222 Rosewood Drive, Danvers, MA 01923; include the code 0022-4650/ 11 and $\$ 10.00$ in correspondence with the CCC.

${ }^{*}$ Aerospace Engineer, Vehicle Analysis Branch. Member AIAA.

${ }^{\dagger}$ Aerospace Engineer, Vehicle Analysis Branch. Senior Member AIAA.

${ }^{\ddagger}$ Senior Project Engineer. Member AIAA.

${ }^{\S}$ Aerospace Engineer, Vehicle Analysis Branch. Associate Fellow AIAA.

$$
\begin{aligned}
& \mathbf{x}_{A}, \mathbf{x}_{B} \\
& \ddot{\mathbf{x}}_{A}, \ddot{\mathbf{x}}_{B} \\
& \alpha \quad=\text { angle of attack, deg } \\
& \beta \quad=\text { angle of sideslip, deg } \\
& \Delta x, \Delta y, \Delta z=x, y \text {, and } z \text { components of joint translational } \\
& \text { displacement in local body frame, } \mathrm{ft} \\
& \eta \quad=\text { Baumgarte error control parameter } \\
& \rho_{A}, \rho_{B} \quad=\text { position vector from point } A \text { mass center to } \\
& \text { body } \bar{A} \text { and body } B \text { mass center to point } \bar{B} \text {, ft } \\
& \omega_{A}, \omega_{B} \quad=\text { angular velocity vector of body } A \text { and body } \\
& \omega_{x}, \omega_{y}, \omega_{z} \quad=\text { body components of inertial angular velocity, } \\
& \dot{\omega}_{A}, \dot{\omega}_{B}
\end{aligned}
$$

\section{Introduction} NALYZING the dynamic separation of multiple bodies within
the atmosphere is complex and challenging. One problem that
has received significant attention in the literature is that of store
separation from aircraft [1]. A similar example is the separation of the
X-15 research vehicle (RV) from the B-52 carrier aircraft [2]. In both
of these cases, the store and the X-15 vehicle are much smaller in size
than the parent vehicle. The other class of stage separation problem
involves separation of two vehicles of comparable sizes, as in the case
of multistage reusable launch vehicles, where the integrity of each
stage is important after separation.
NASA studies on stage separation of multistage reusable launch
vehicles date back to the early 1960s [3-7]. These studies addressed
the problem of separation of generic two-stage reusable launch
vehicles. More recently, Naftel and Wilhite [8] and Naftel and Powell
[9,10] considered staging of dual wing-body vehicles. NASA's
interest in stage separation research was renewed in early 2000 in
connection with design and development of multistage launch
vehicles. Accordingly, NASA initiated a comprehensive stage
separation tool development activity that included wind-tunnel
testing as well as development and validation of computational fluid
dynamic and engineering-level simulation tools [11]. As a part of this 
activity, a stage separation analysis and simulation tool called ConSep (short for conceptual separation) was developed, which is a front end to the commercially available multibody dynamic analysis software ADAMS ${ }^{\circledR}$ [12]. References [13-15] discuss the application of ConSep to two-body and three-body separation problems. Because it is tied to ADAMS, one disadvantage of ConSep is that it cannot be easily integrated into standard trajectory simulation software for performing efficient and seamless end-to-end simulations of launch vehicle trajectories.

The objective of this paper is to discuss application of the constraint force equation (CFE) methodology [16] for multibody stage separation and its implementation in the Program to Optimize Simulated Trajectories II (POST2) [17,18] for efficient, seamless end-to-end simulation of launch vehicle trajectories including stage separation. The paper also discusses the verification of the CFE methodology and POST2 implementation using three specifically designed test cases. Even though this paper discusses the CFE implementation in POST2, the method is generic in nature and can be implemented in any similar trajectory program capable of simulating multiple, unconnected vehicles.

By itself, POST2 does not have the capability to model internal joint forces and moments before separation when the bodies are still connected. The CFE implementation in POST2 provides a framework for computing the internal constraint forces and moments acting at joints connecting multiple vehicles. These constraint forces and moments can then be applied to each body, together with the usual external forces and moments due to gravity, aerodynamics, and propulsion. Thus, the CFE methodology simply augments the external loads and does not require modification of the POST2 equations of motion. The CFE methodology provides the missing link to model accurately the dynamics of generic multibody separation.

This paper describes the steps taken to verify and validate the CFE methodology through its application to three test cases of increasing complexity. The verification was performed by comparing results of each test case to results obtained from benchmark codes and, when possible, flight data. The first test case simulates the motion of two rigid bodies connected by a fixed joint, with no external forces. The advantage of studying this basic problem is that numerical results can be compared with what is to be expected on the basis of first principles. The second test case models two rigid bodies connected by a universal joint. The third test case consists of a comparison of a POST2/CFE simulation of the Mach 7 separation of the X-43A vehicle to flight data and to previously published preflight simulation results. These three test cases were part of a larger, comprehensive series of checks that were performed on the CFE methodology to gain confidence in its ability to solve complex constrained motion problems using conventional trajectory programs, such as POST2. For the first two cases, the CFE simulations are in excellent agreement with independent simulations developed using benchmark industry-standard software packages. For the third test case, CFE performs as well or better than the preflight simulation, was easier to implement, and its results compare reasonably well with the flight data.

\section{Constraint Force Equation Methodology}

\section{A. General Formulation}

To illustrate the basic concept of the CFE methodology, consider the motion of two rigid bodies connected by a single joint, as shown in Fig. 1. The external forces and moments that act on each body are shown in Fig. 1a. These external forces $\left(\mathbf{F}_{A}^{(\mathrm{EXT})}\right.$ and $\left.\mathbf{F}_{B}^{(\mathrm{EXT})}\right)$ and external torques, or moments of force about mass centers $\left(\mathbf{T}_{A}^{(\mathrm{EXT})}\right.$ and $\mathbf{T}_{B}^{(\mathrm{EXT})}$ ), are the resultants of gravity, aerodynamic, and propulsive forces or moments on each vehicle. Figure $1 \mathrm{~b}$ shows the internal constraint forces and moments $\left(\mathbf{F}^{(\mathrm{CON})}\right.$ and $\left.\overline{\mathbf{T}}^{(\mathrm{CON})}\right)$ acting on each vehicle at the joint location. These internal forces and moments constrain the way in which one vehicle can move relative to the other, and they are dependent upon the external forces acting on each vehicle as well as the type of joint. For joints that permit only relative rotation between two bodies, as shown in Fig. $\underline{1 b}$, the forces and moments on one body have magnitudes that are equal and directions that are opposite to those acting on the other body. The objective of the CFE methodology is to compute these internal forces and moments and apply them as external forces and moments on each body. Figure 1c illustrates the way in which CFE is implemented in POST2. At each integration time step, the current version of POST2 computes the typical external forces and moments acting on each vehicle. This information, along with specific geometric information about the joint, is provided to the CFE routine, which works in parallel to compute the internal forces and moments required to satisfy the constraints imposed by the joint. Next, these internal joint loads are applied to each vehicle as additional external forces and moments, and the POST2 solution is propagated in the usual manner to the next time step. Thus, the net external forces and moments on each vehicle are the sum of 1) the usual external forces and moments and 2) the joint loads applied to each vehicle as additional external forces and moments. Consequently, the CFE joint model simply augments the vehicle external loads and does not require modification to the POST2 equations of motion.

The equations of constrained motion of two rigid bodies ( $A$ and $B$ ) connected by a single joint (point $\bar{A}$ in body $A$ and point $\bar{B}$ in body $B$ ) are as follows. For body $A$,

$$
\mathbf{F}_{A}^{(\mathrm{EXT})}+\mathbf{F}_{A}^{(\mathrm{CON})}=m_{A} \ddot{\mathbf{x}}_{A}
$$

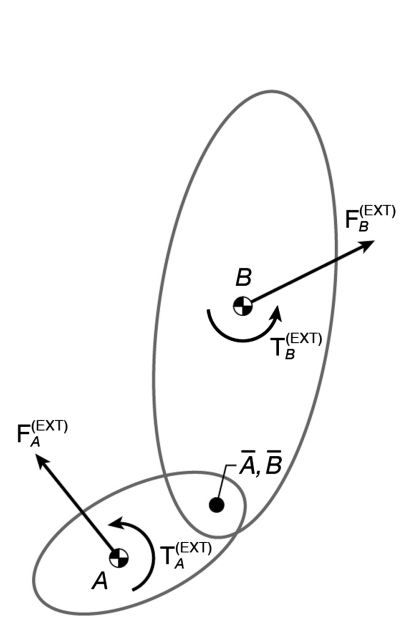

a) External forces and moments

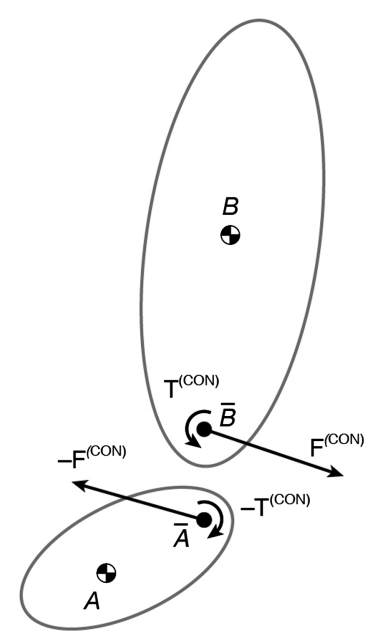

b) Internal forces and moments

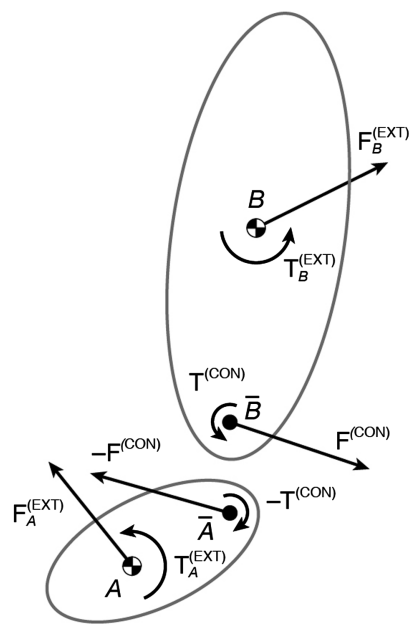

c) Resultant forces and moments

Fig. 1 Illustration of basic concept of CFE methodology. 


$$
\mathbf{T}_{A}^{(\mathrm{EXT})}+\boldsymbol{\rho}_{A} \times \mathbf{F}_{A}^{(\mathrm{CON})}+\mathbf{T}_{A}^{(\mathrm{CON})}=\mathbf{I}_{A} \cdot \dot{\boldsymbol{\omega}}_{A}+\boldsymbol{\omega}_{A} \times \mathbf{I}_{A} \cdot \boldsymbol{\omega}_{A}
$$

where $\rho_{A}$ is the position vector from the mass center of $A$ to point $\bar{A}$ of $A$ at which the constraint force is applied. Similarly, for body $B$,

$$
\begin{gathered}
\mathbf{F}_{B}^{(\mathrm{EXT})}+\mathbf{F}_{B}^{(\mathrm{CON})}=m_{B} \ddot{\mathbf{x}}_{B} \\
\mathbf{T}_{B}^{(\mathrm{EXT})}+\boldsymbol{\rho}_{B} \times \mathbf{F}_{B}^{(\mathrm{CON})}+\mathbf{T}_{B}^{(\mathrm{CON})}=\mathbf{I}_{B} \cdot \dot{\boldsymbol{\omega}}_{B}+\boldsymbol{\omega}_{B} \times \mathbf{I}_{B} \cdot \boldsymbol{\omega}_{B}
\end{gathered}
$$

Note that, in the case of unconstrained (free) motion, the constraint forces and moments are equal to zero, and Eqs. (1-4) reduce to the standard equations of motion for two free rigid bodies. Each mass center acceleration, rigid body angular acceleration, constraint force, and constraint torque are regarded as unknowns; therefore, Eqs. (14) represent 12 scalar equations in 24 unknowns. Hence, 12 additional scalar equations are required. Of these, six are obtained from two vector relationships that are consequences of the law of action and reaction:

$$
\begin{gathered}
\mathbf{F}_{A}^{(\mathrm{CON})}+\mathbf{F}_{B}^{(\mathrm{CON})}=\mathbf{0} \\
\mathbf{T}_{A}^{(\mathrm{CON})}+\mathbf{T}_{B}^{(\mathrm{CON})}+\left(\mathbf{r}_{B}-\mathbf{r}_{A}\right) \times \mathbf{F}_{B}^{(\mathrm{CON})}=\mathbf{0}
\end{gathered}
$$

where $\mathbf{r}_{A}$ is the position vector from a point fixed in an inertial reference frame to $\bar{A}$. Because $\mathbf{x}_{A}$ is the position vector from a point fixed in an inertial reference frame to the mass center of $A$, we can write $\mathbf{r}_{A}=\mathbf{x}_{A}+\boldsymbol{\rho}_{A}$. Note that vector $\boldsymbol{\rho}_{A}$ is fixed in $A$. Position vector $\mathbf{r}_{B}$ is defined similarly.

The six remaining equations fall into one of two categories. First, equations of constraint are formed that describe how a particular joint restricts relative translation or relative rotation. Second, by considering the translation or rotation that is permitted by the joint, equations are written to account for the absence of constraint force or torque in a particular direction in view of the ideal (perfectly smooth) nature of the joint.

When relative translation is constrained, the distance between two points must remain fixed in a particular direction. That is,

$$
\left(\mathbf{r}_{B}-\mathbf{r}_{A}\right) \cdot \mathbf{e}_{A}=0
$$

where the joint prevents relative translation in the direction of unit vector $\mathbf{e}_{A}$, which is fixed in body $A$. One equation having the form of Eq. (7) is needed to account for each direction in which translation is constrained. For example, a fixed joint constrains translation in three orthogonal directions; therefore, three equations in the form of Eq. (7) are required. In each such equation, the role of $\mathbf{e}_{A}$ is played by one of three mutually orthogonal unit vectors fixed in $A$. For a prismatic (sliding) joint that permits translation in only one direction (and restricts translation in two perpendicular directions), two equations having the form of Eq. (7) are required.

A constraint on relative rotation can be viewed as a requirement that two unit vectors must remain perpendicular; each unit vector is fixed in one of the bodies and is normal to the axis about which rotation would take place if the constraint were not present. The constraint is expressed by setting the scalar product of the two unit vectors equal to zero:

$$
\mathbf{e}_{B} \cdot \mathbf{e}_{A}=0
$$

where the two unit vectors, $\mathbf{e}_{A}$ and $\mathbf{e}_{B}$ (one fixed in each body), are chosen to be perpendicular to each other throughout the constrained motion. One equation in the form of Eq. (8) is required for each direction about which rotation is constrained.

In their current form, Eqs. (ㄱ) and ()ㅡㄹ are not coupled with Eqs. (14). For this purpose, the constraint equations (7) and (8) are differentiated twice with respect to time in an inertial reference frame so that the resulting equations involve the unknown linear and angular accelerations of bodies $A$ and $B$, and thus can be coupled to the equations of motion. To this end, the second derivatives that need to be evaluated are

$$
\begin{gathered}
\frac{\mathrm{d}^{2}}{\mathrm{~d} t^{2}}\left[\left(\mathbf{r}_{B}-\mathbf{r}_{A}\right) \cdot \mathbf{e}_{A}=0\right] \\
\frac{\mathrm{d}^{2}}{\mathrm{~d} t^{2}}\left[\mathbf{e}_{B} \cdot \mathbf{e}_{A}=0\right]
\end{gathered}
$$

The result of differentiating Eq. (7) once is

$$
\left(\dot{\mathbf{r}}_{B}-\dot{\mathbf{r}}_{A}\right) \cdot \mathbf{e}_{A}+\left(\mathbf{r}_{B}-\mathbf{r}_{A}\right) \cdot\left(\omega_{A} \times \mathbf{e}_{A}\right)=0
$$

where $\dot{\mathbf{r}}_{A}$ and $\dot{\mathbf{r}}_{B}$ are, respectively, the time derivatives in an inertial reference frame of $\mathbf{r}_{A}$ and $\mathbf{r}_{B}$. Differentiating once more, one obtains

$$
\begin{aligned}
& \left(\ddot{\mathbf{x}}_{B}+\dot{\omega}_{B} \times \rho_{B}-\ddot{\mathbf{x}}_{A}-\dot{\omega}_{A} \times \rho_{A}\right) \cdot \mathbf{e}_{A}+\left(\mathbf{r}_{B}-\mathbf{r}_{A}\right) \cdot\left(\dot{\omega}_{A} \times \mathbf{e}_{A}\right) \\
& \quad=2\left(\dot{\mathbf{r}}_{B}-\dot{\mathbf{r}}_{A}\right) \cdot\left(\mathbf{e}_{A} \times \omega_{A}\right)-\left(\mathbf{r}_{B}-\mathbf{r}_{A}\right) \cdot\left(\omega_{A} \times \omega_{A} \times \mathbf{e}_{A}\right) \\
& \quad+\left[\left(\omega_{A} \times \omega_{A} \times \rho_{A}\right)-\left(\omega_{B} \times \omega_{B} \times \rho_{B}\right)\right] \cdot \mathbf{e}_{A}
\end{aligned}
$$

Differentiating once with respect to time in Eq. (ㅁ) yields

$$
\left(\omega_{B} \times \mathbf{e}_{B}\right) \cdot \mathbf{e}_{A}+\mathbf{e}_{B} \cdot\left(\omega_{A} \times \mathbf{e}_{A}\right)=0
$$

and differentiating once more gives

$$
\begin{aligned}
& \left(\dot{\omega}_{B}-\dot{\omega}_{A}\right) \cdot\left(\mathbf{e}_{B} \times \mathbf{e}_{A}\right)=\left(\omega_{B}-\omega_{A}\right) \cdot\left[\mathbf{e}_{A} \times\left(\omega_{B} \times \mathbf{e}_{B}\right)\right. \\
& \left.-\mathbf{e}_{B} \times\left(\omega_{A} \times \mathbf{e}_{A}\right)\right]
\end{aligned}
$$

When relative translation is permitted in a certain direction, an equation having the form of Eq. (12) is not required. Likewise, when relative rotation is allowed, a relationship in the form of Eq. (14) is not necessary. Instead, the required equations can be obtained using the condition that the constraint forces or moments are zero in those directions (with the assumption that the surfaces of the joint are perfectly smooth). Thus, for a joint that permits translation in a certain direction,

$$
\mathbf{F}^{(\mathrm{CON})} \cdot \mathbf{e}=0
$$

Depending on the circumstances, $\mathbf{F}^{(\mathrm{CON})}$ can be the constraint force acting either on $A$ or on $B$. Similarly, when a joint permits relative rotation about a certain direction,

$$
\mathbf{T}^{(\mathrm{CON})} \cdot \mathbf{e}=0
$$

In summary, equations having the form of Eqs. (12) and (14-16) provide, in combination, a total of six scalar equations.

\section{B. Joint Stabilization}

Equations (1-16) represent a sufficient set for solving the general constraint load problem described herein. However, the accuracy of the constraint load solution is sensitive to computational numerical error and initial joint misalignment. In particular, an accumulation of numerical error typically manifests itself as joint separation and/or misalignment error over time. This in turn introduces additional errors in the solution, and the cycle continues, leading to a buildup of error over time. To reduce, or even reverse, joint separation and misalignment errors, the CFE algorithm implements an optional stabilization technique known as Baumgarte [19] stabilization. Ordinarily, the constraint equations (12) and (14) take the following generalized form:

$$
\ddot{g}=0
$$

where $g$ represents either of the (nondifferentiated) constraints in Eqs. (7) and (8). The stabilization technique is implemented by augmenting Eq. (17) with terms involving the once differentiated and nondifferentiated forms of $g$ :

$$
\ddot{g}+2 \eta \dot{g}+\eta^{2} g=0
$$


Thus, the new constraint relation in Eq. (18) considers not only the acceleration constraints but also the corresponding velocity and original constraints. These additional terms serve as a form of proportional and derivative control that can mitigate joint separation and misalignment errors. Since the stabilization effect is dependent on the specific dynamic system, the Baumgarte parameter $\eta$ can be tuned to control the degree of constraint error for a particular application.

\section{Implementation}

To implement the CFE method for a single joint connecting two rigid bodies, 24 scalar equations are formed from Eqs. (1-6), (12), and (14-16) that are linear in 24 unknowns. These equations can be expressed in matrix form, $A x=b$, and solved accordingly using a standard matrix inversion technique. The column matrix $x$ contains the 24 unknown parameters, which are the scalar components of the following vectors: $\ddot{\mathbf{x}}_{A}, \ddot{\mathbf{x}}_{B}, \dot{\boldsymbol{\omega}}_{A}, \dot{\boldsymbol{\omega}}_{B}, \mathbf{F}_{A}^{(\mathrm{CON})}, \mathbf{F}_{B}^{(\mathrm{CON})}, \mathbf{T}_{A}^{(\mathrm{CON})}$, and $\mathbf{T}_{B}^{(\mathrm{CON})}$. Matrix $A$ can be singular when, for example, one neglects a central principal moment of inertia, such as for a slender rod, or when the mass of a body is neglected. The matrix also becomes singular when constraint equations are redundant; in other words, when one is linearly dependent on the others. An example of a redundant constraint equation would be a body connected by two joints that both constrain translation in a given direction. Thus, care should be taken to formulate the problem in a way that avoids these difficulties. The X-43A test case discussed in Sec. III.C explains how the problem's formulation was altered in order to avoid redundant constraint equations.

In general, the CFE method can be applied to problems containing multiple bodies and multiple joints, so long as there are not redundant constraints. In general, the number $N$ of equations and unknowns involved in a problem is given by

$$
N=6\left(N_{B}+N_{j}\right)
$$

where $N_{B}$ is the number of rigid bodies, and $2 N_{j}$ is the number of joints.

Finally, it is possible to improve computational efficiency by implementing the CFE algorithm in a way that reduces the number of equations and unknowns. One technique that has been successfully employed by the authors is the conventional minimal matrix approach [20-22]. This technique combines Eqs. (1), (2), (12), and (14) to solve only for the unknown constraint loads rather than solving for $N$ unknowns. The minimal matrix approach is advantageous, since it involves only those constraint equations that are needed for any particular joint configuration; thus, no extraneous calculations are made.

\section{Test Cases}

A suite of test cases was developed to check and verify the CFE methodology for application to multibody separation problems in the launch vehicle staging environment. These test cases are listed in Table 1 and cover the types of joints typically needed to model stage separation problems. The list is not exhaustive and, in general, it is possible to model joints that simultaneously constrain any

Table 1 Test cases performed for CFE verification (2-D denotes two-dimensional)

\begin{tabular}{llcc}
\hline \hline Case & \multicolumn{1}{c}{ Joint type } & $\begin{array}{c}\text { Translational } \\
\text { degrees of } \\
\text { freedom }\end{array}$ & $\begin{array}{c}\text { Rotational } \\
\text { degrees of } \\
\text { freedom }\end{array}$ \\
\hline 1 & Fixed & 0 & 0 \\
2 & Revolute (hinge) & 0 & 1 \\
3 & Universal & 0 & 2 \\
4 & Ball & 0 & 3 \\
5 & 1-D translational (slider) & 1 & 0 \\
6 & 2-D translational (planar) & 2 & 0 \\
7 & Cylindrical & 1 & 1 \\
\hline \hline
\end{tabular}

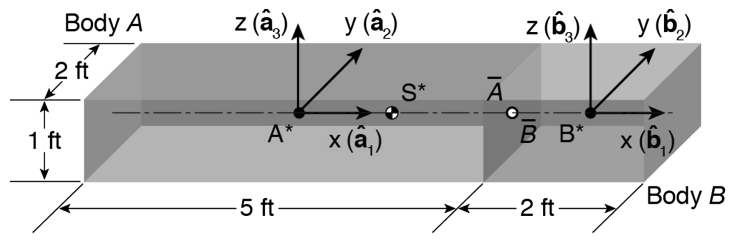

Fig. 2 Test case 1: two rigid bodies, $A$ and $B$, connected by a fixed joint.

combination of translational and rotational degrees of freedom with CFE simply by forming the appropriate equations described in Eqs. (12) and (14-16).

A description of the use of CFE to solve test cases 2-7 from Table 1 can be found in [23]. Two test cases from Table 1 (the fixed joint and the universal joint) are discussed in this paper to illustrate the testing and verification that was performed on the CFE methodology. A third test case was conducted that used POST2/CFE to model the X-43A stage separation problem as a one-dimensional (1-D) translational (or sliding) joint in series with a revolute (or hinge) joint.

\section{A. Test Case 1: Fixed Joint}

This test case involves two rigid bodies, denoted as bodies $A$ and $B$, connected by a fixed joint (see Fig. 2). The mass properties for each body are shown in Table 2 . The fixed joint constrains point $\bar{A}$ attached in $A$ to remain coincident with point $\bar{B}$ attached in $B$. Three constraint equations having the form of Eq. (7) can be written as

$$
\left(\mathbf{r}_{B}-\mathbf{r}_{A}\right) \cdot \hat{\mathbf{a}}_{r}=0 \quad(r=1,2,3)
$$

where the role of $\mathbf{e}_{A}$ in Eq. (7) is played in turn by $\hat{\mathbf{a}}_{1}, \hat{\mathbf{a}}_{2}$, and $\hat{\mathbf{a}}_{3}$, each of which belongs to a set of three right-handed mutually orthogonal unit vectors fixed in $A$, as shown in Fig. 2 . Suppose that $\hat{\mathbf{b}}_{1}, \hat{\mathbf{b}}_{2}$, and $\hat{\mathbf{b}}_{3}$ are a similar set of unit vectors fixed in $B$, such that $\hat{\mathbf{b}}_{r}$ has the same direction as $\hat{\mathbf{a}}_{r}(r=1,2,3)$ when $A$ and $B$ are attached to each other. In that case, the fixed joint constrains certain unit vectors fixed in $A$ to remain perpendicular to other unit vectors fixed in $B$. For example, one can write the following three constraint equations having the form of Eq. (ㅁ):

$$
\begin{aligned}
& \hat{\mathbf{b}}_{2} \cdot \hat{\mathbf{a}}_{1}=0 \\
& \hat{\mathbf{b}}_{3} \cdot \hat{\mathbf{a}}_{1}=0 \\
& \hat{\mathbf{b}}_{2} \cdot \hat{\mathbf{a}}_{3}=0
\end{aligned}
$$

Thus, a total of six constraint equations have been written. As mentioned previously, these constraint equations are differentiated twice with respect to time so that the resulting relationships involve the unknown accelerations and angular accelerations. Thus, one employs three equations having the form of Eq. (12), in which the role of $\mathbf{e}_{A}$ is played by $\hat{\mathbf{a}}_{1}, \hat{\mathbf{a}}_{2}$, and $\hat{\mathbf{a}}_{3}$, respectively. Likewise, three equations having the form of Eq. (14) come into play. In the first of these, the roles of $\mathbf{e}_{A}$ and $\mathbf{e}_{B}$ are played by $\hat{\mathbf{a}}_{1}$ and $\hat{\mathbf{b}}_{2}$. In the second such equation, $\hat{\mathbf{a}}_{1}$ and $\hat{\mathbf{b}}_{3}$ play the parts of $\mathbf{e}_{A}$ and $\mathbf{e}_{B}$. In the third relationship, $\hat{\mathbf{a}}_{3}$ and $\hat{\mathbf{b}}_{2}$ are substituted for $\mathbf{e}_{A}$ and $\mathbf{e}_{B}$.

No relationships having the form of Eq. (15) or Eq. (16) are applicable in the case of the fixed joint, because no relative motion is permitted by the joint.

Table 2 Mass properties for test case 1, fixed joint

\begin{tabular}{lcc}
\hline \hline & Body $A$ & Body $B$ \\
\hline$m$ & 1 slug & $4 / 10 \mathrm{slug}$ \\
$I_{x x}$ & $5 / 12 \mathrm{slug} \cdot \mathrm{ft}^{2}$ & $1 / 6$ slug $\cdot \mathrm{ft}^{2}$ \\
$I_{y y}$ & $26 / 12 \mathrm{slug} \cdot \mathrm{ft}^{2}$ & $1 / 6$ slug $\cdot \mathrm{ft}^{2}$ \\
$I_{z z}$ & $29 / 2 \mathrm{slug} \cdot \mathrm{ft}^{2}$ & $4 / 15 \mathrm{slug} \cdot \mathrm{ft}^{2}$ \\
\hline \hline
\end{tabular}




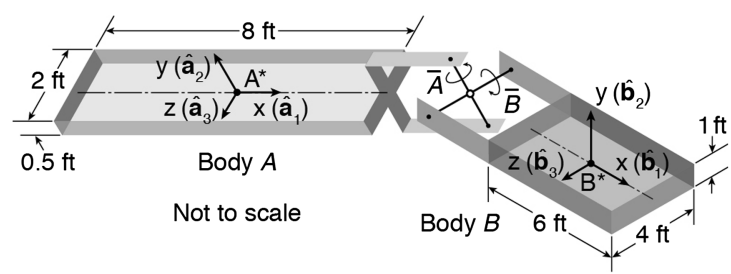

Fig. 3 Test Case 2: Two rigid bodies, $A$ and $B$, connected by a universal joint.

\section{B. Test Case 2: Universal Joint}

This test case involves two rigid bodies, $A$ and $B$, for which the mass is distributed uniformly, and they are connected by a universal joint. The vector from the joint to $A^{*}$ is $-4.854 \hat{\mathbf{a}}_{1}-0.096 \hat{\mathbf{a}}_{2}$, and the vector from the joint to $B^{*}$ is $3.851 \hat{\mathbf{b}}_{1}-0.096 \hat{\mathbf{b}}_{2}$. This configuration is shown in Fig. 3 . The universal joint constrains point $\bar{B}$ fixed in $B$ to remain coincident with point $\bar{A}$ fixed in $A$ at the intersection of the two arms in the cross. Therefore, the three translational constraint equations presented in Eq. (20) for the fixed joint apply in this case as well.

For this problem, one rotational constraint equation having the form of Eq. ( $\underline{\text { ) }}$ is needed. If $\hat{\mathbf{a}}_{2}$ is chosen to be parallel to the arm that is fixed in $A$, and $\hat{\mathbf{b}}_{3}$ is chosen to be parallel to the arm that is fixed in $B$, then the rotational constraint equation imposed by the universal joint can be described with the relationship

$$
\hat{\mathbf{b}}_{3} \cdot \hat{\mathbf{a}}_{2}=0
$$

The foregoing four constraint equations are differentiated twice with respect to time. Thus, for the translational constraint, one employs three equations having the form of Eq. (12), in which the role of $\mathbf{e}_{A}$ is played by $\hat{\mathbf{a}}_{1}, \hat{\mathbf{a}}_{2}$, and $\hat{\mathbf{a}}_{3}$, respectively. Likewise, for the rotational constraint, the result is obtained by employing Eq. (14) and making two substitutions: $\hat{\mathbf{a}}_{2}$ for $\mathrm{e}_{A}$, and $\hat{\mathbf{b}}_{3}$ for $\mathbf{e}_{B}$.

The other two equations are obtained with the aid of Eq. (16): it is assumed that the universal joint is ideal (frictionless), so that no constraint torque is exerted in the directions of the arms in the cross:

$$
\mathbf{T}_{A}^{(\mathrm{CON})} \cdot \hat{\mathbf{a}}_{2}=0, \quad \mathbf{T}_{B}^{(\mathrm{CON})} \cdot \hat{\mathbf{b}}_{3}=0
$$

The mass properties of each body are summarized in Table $\underline{3}$ (products of inertia are zero for each body in this problem).

\section{Test Case 3: $\mathrm{X}-\mathbf{4 3 A}$}

This test case was selected to evaluate the capability of POST2/ CFE to model and simulate a realistic stage separation problem by comparing results with flight data from NASA's X-43A hypersonic scramjet test vehicle (see Fig. 4). In 2004, NASA conducted two successful scramjet test flights of the X-43A RV at speeds near Mach 7 and 10. The roughly $10 \mathrm{~s}$ scramjet test was performed after the RV was boosted to the target flight condition by the Hyper-X launch vehicle (HXLV). A dimensioned drawing of the mated RV and HXLV is shown in Fig. 5. Additional information describing the X-43A RV and HXLV may be found in [24].

The X-43A stage separation event was initiated approximately $3 \mathrm{~s}$ after HXLV burnout at a dynamic pressure of 1000 psf. Separation began when two pyrotechnically actuated pistons extending from the booster pushed against the $\mathrm{RV}$ to induce $\sim 16 \mathrm{ft} / \mathrm{s}$ of relative velocity between the two vehicles. The two pistons, which were positioned

Table 3 Mass properties for test case 2, universal joint

\begin{tabular}{lcc}
\hline \hline & Body $A$ & Body $B$ \\
\hline$m$ & 622.0 slugs & 622.0 slugs \\
$I_{x x}$ & 220.3 slug $\cdot \mathrm{ft}^{2}$ & 881.2 slug $\cdot \mathrm{ft}^{2}$ \\
$I_{y y}$ & 3524.7 slug $\cdot \mathrm{ft}^{2}$ & 2695.3 slug $\cdot \mathrm{ft}^{2}$ \\
$I_{z z}$ & 3330.3 slug $\cdot \mathrm{ft}^{2}$ & 1917.8 slug $\cdot \mathrm{ft}^{2}$ \\
\hline \hline
\end{tabular}

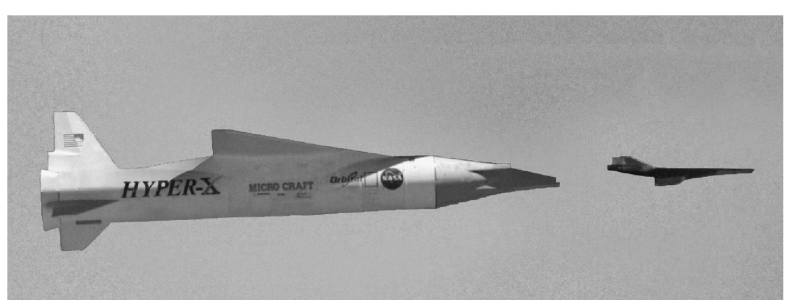

Fig. 4 Test case 3: artistic rendering of X-43A (RV) separation from HXLV booster.

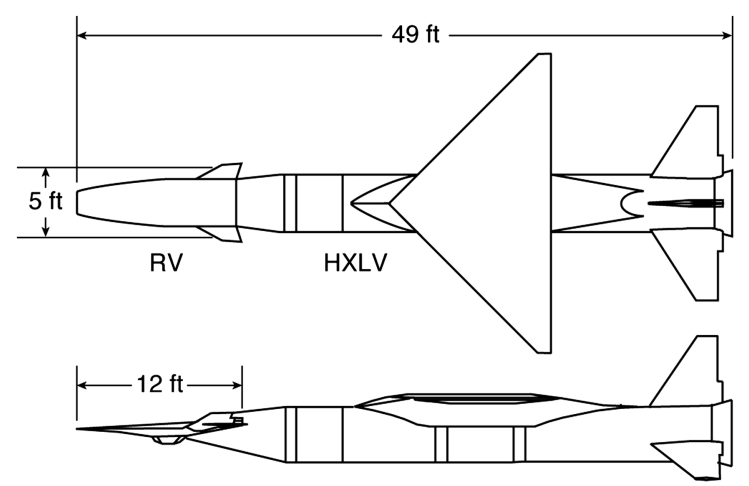

Fig. 5 Test case 3: dimensioned drawing of mated X-43A RV and HXLV.

roughly 9 in. on each side of the RV centerline, were initially in contact with a cuplike ball joint attached to the RV that permitted rotation about the piston contact point and translation only along the line of action of the piston force, which was oriented 4 deg below the horizontal. Both pistons were connected to the same gas chamber and were activated at the same time and remained in contact with the RV for $\sim 0.1 \mathrm{sec}$ until they reached the end of their 9 in. stroke length. Although the ball joint permitted rotation in any direction at each individual piston contact point, the fact that both pistons were fired concurrently and were simultaneously in contact with the RV effectively excluded relative rolling and yawing rotation between the two separating vehicles.

Once the pistons reached the end of their 9 in. extensions, the RV separated from the HXLV. At this time, the HXLV commanded a nosedown pitching moment to move away from the RV, and the RV was steered to its required target condition.

To test the CFE methodology, this problem was set up using POST2/CFE to model the piston constraint forces. For the purpose of this paper, the two pistons were modeled as a single piston to avoid overconstraining the problem. Thus, a single piston was modeled as a sliding joint in series with a revolute joint; that is, relative translation was allowed in one direction (the piston line of action) and relative rotation was permitted only about the pitch axis. The piston was placed at the centerline of the RV, and the axial force that pushed the vehicles apart was doubled. A schematic highlighting these modeling details is shown in Fig. 6.

To illustrate how the constraint equations for this joint are derived, unit vectors are defined in the HXLV such that $\hat{\mathbf{a}}_{1}$ is the direction in which translation or sliding is permitted (along the piston line of action) and $\hat{\mathbf{a}}_{2}$ indicates the direction in which rotation is free to occur (pitch axis). A similar set of unit vectors $\hat{\mathbf{b}}_{r}$ can be defined that are fixed in the RV and initially aligned with the unit vectors $\hat{\mathbf{a}}_{r}$ fixed in the HXLV (see Fig. 6). For this case, two constraint equations having the form of Eq. (7) can be written as

$$
\left(\mathbf{r}_{B}-\mathbf{r}_{A}\right) \cdot \hat{\mathbf{a}}_{r}=0 \quad(r=2,3)
$$

As before, these two constraint equations are differentiated twice with respect to time, as in Eq. (12). Now, $\hat{\mathbf{a}}_{2}$ and $\hat{\mathbf{a}}_{3}$ are substituted for $\mathbf{e}_{A}$. Furthermore, two constraint equations describing the restriction imposed on relative orientation are formed: 


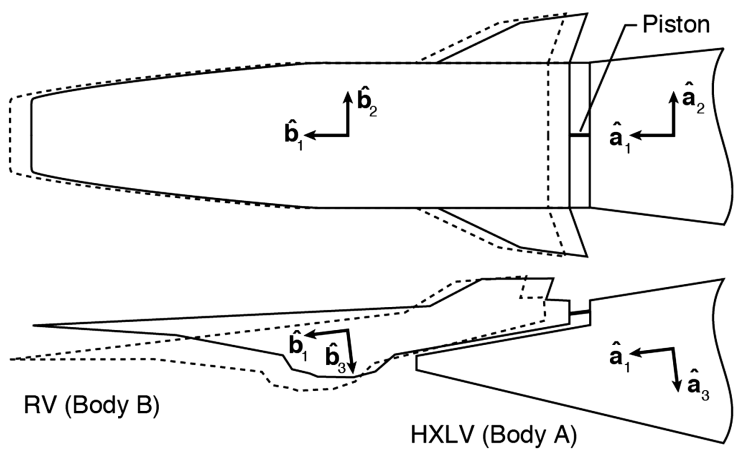

Fig. 6 Test case 3: CFE modeling of piston contact for $\mathrm{X}-43 \mathrm{~A}$ separation problem.

$$
\hat{\mathbf{b}}_{1} \cdot \hat{\mathbf{a}}_{2}=0, \quad \hat{\mathbf{b}}_{3} \cdot \hat{\mathbf{a}}_{2}=0
$$

Similarly, these constraint equations are differentiated twice with respect to time, as in Eq. (14), where $\hat{\mathbf{a}}_{2}$ is now substituted for $\mathbf{e}_{A}$, and $\hat{\mathbf{b}}_{1}$ and $\hat{\mathbf{b}}_{3}$ are substituted for $\mathbf{e}_{B}$. With the assumption that the joint is frictionless, the constraint force and moment in the directions where motion is permitted become, using Eqs. (15) and (16),

$$
\mathbf{F}_{B}^{(\mathrm{CON})} \cdot \hat{\mathbf{a}}_{1}=0, \quad \mathbf{T}_{B}^{(\mathrm{CON})} \cdot \hat{\mathbf{a}}_{2}=0
$$

\section{Results and Discussion}

\section{A. Test Case I: Fixed Joint}

The two rigid bodies, $A$ and $B$, are assumed to be rigidly connected to each other for $10 \mathrm{~s}$ (see Fig. 2) and then released instantaneously. No external forces or moments were assumed to act on either body for the entire duration of the $20 \mathrm{~s}$ simulation. Initial conditions correspond to zero inertial velocity of the system mass center, $S^{*}$, and an inertial angular velocity of the rigid body formed by $A$ and $B$ equal to

$$
63.02 \hat{\mathbf{a}}_{1}-82.32 \hat{\mathbf{a}}_{2}+80.25 \hat{\mathbf{a}}_{3} \mathrm{deg} / \mathrm{s}
$$

After $10 \mathrm{~s}$, the joint connecting $A$ and $B$ is released, and their motions become unconstrained. Because there are no external forces or moments, integrals of the motion involving linear and angular momentum must remain constant, and this fact can be used to check numerical solutions of the equations of motion. Therefore, one can expect the simulation results to show that, after separation, the mass centers of $A$ and $B$ travel in straight lines with constant velocities.

To verify the POST2/CFE simulation results, a simulation was also created with AUTOLEV [25-27], an interactive program designed specifically for the kinematic and dynamic analysis of mechanical systems. Symbolic manipulation (computer algebra) is used to formulate explicit equations of motion for the particular system of interest using any method; the software is particularly well suited to Kane's method $[27,28]$. For this test case, AUTOLEV was used to create a computer program to simulate the motions of $A$ and $B$ and determine the constraint forces required to hold them together for the first $10 \mathrm{~s}$.

The AUTOLEV results were generated using a variable-step integrator with an absolute error limit of $1 \times 10^{-8}$ and a relative error limit of $1 \times 10^{-7}$. The POST2/CFE method employed a fixed-step integrator with a step size of $0.0001 \mathrm{~s}$. The CFE routine also used a Baumgarte parameter of 5.0.

As expected, both linear and angular momentums remained constant throughout the simulation. Time histories for the inertial $x$, $y$, and $z$ components of velocity of the mass center of body $A$ are shown for $20 \mathrm{~s}$ in Fig. 7. The values oscillate during the first $10 \mathrm{~s}$, after which the joint is released and their values become constant. Figure 7 shows that there is an excellent agreement between POST2/CFE and AUTOLEV results. Similarly, the velocity components for the mass center of body $B$ in Fig. $\underline{8}$ also indicate an excellent agreement between POST2/CFE and ĀUTOLEV.

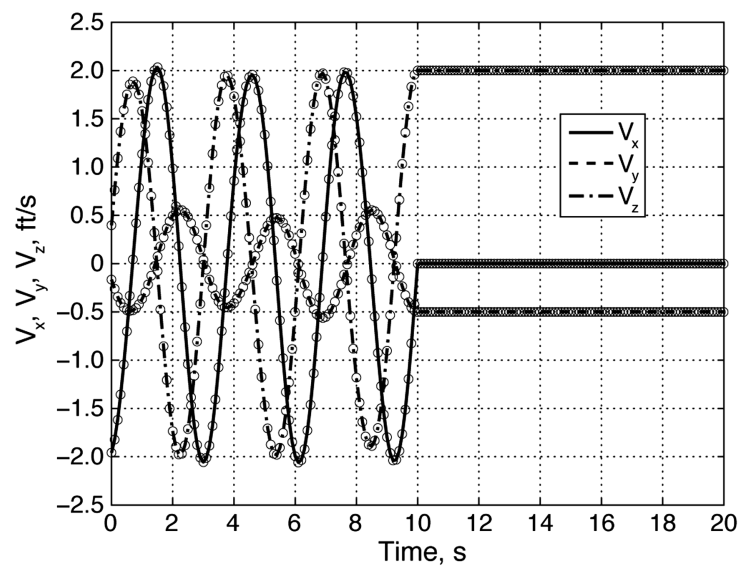

Fig. 7 Test case 1, body $A$ : velocity components of mass center. Joint release occurs at $t=10 \mathrm{~s}$ (circles: POST2/CFE; lines: AUTOLEV).

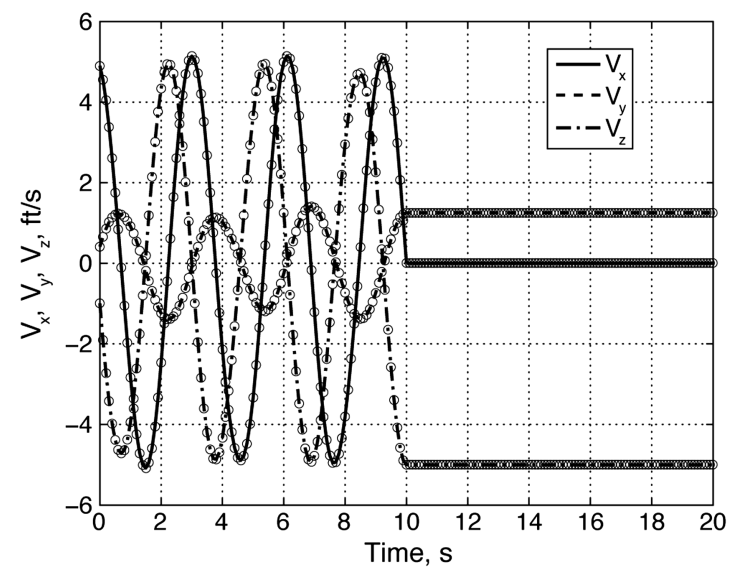

Fig. 8 Test case 1, body $B$ : velocity components of mass center. Joint release occurs at $t=10 \mathrm{~s}$ (circles: POST2/CFE; lines: AUTOLEV).

The angular velocity time history comparisons for bodies $A$ and $B$ are shown in Figs. $\underline{9}$ and $\underline{10}$, respectively. Again, there is excellent agreement between the two approaches. The change in the angular velocity of body $B$ at the time of release is more noticeable than for body $A$, because $B$ is smaller and has a mass distribution that differs from that of the composite body before joint release. The main results of the CFE method are the constraint forces and moments, which are shown in Figs. 11 and 12 and are applied as additional external loads within POST2. The figures show that the forces and torques determined by CFE agree very well with the AUTOLEV results.

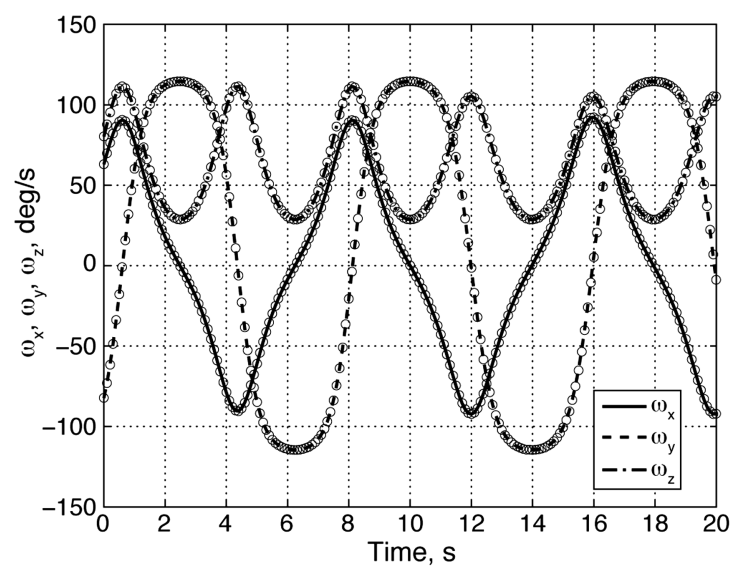

Fig. 9 Test case 1 , body $A$ : angular velocity components. Joint release occurs at $t=10 \mathrm{~s}$ (circles: POST2/CFE; lines: AUTOLEV). 


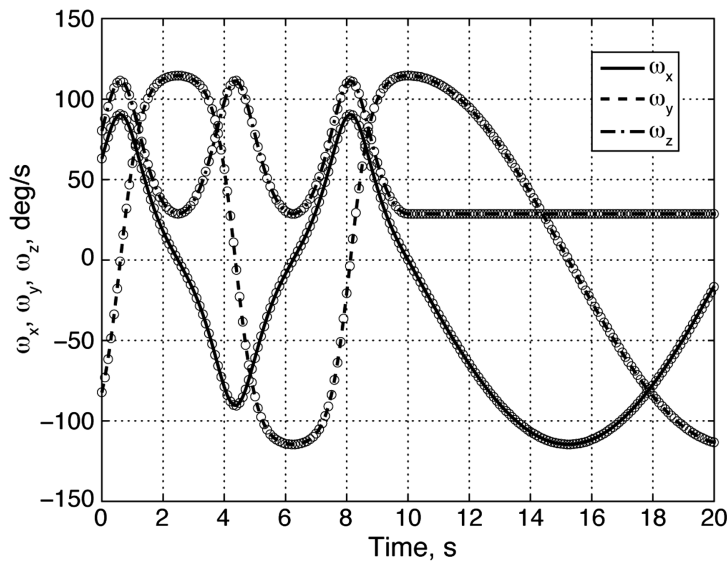

Fig. 10 Test case 1 , body $B$ : angular velocity components. Joint release occurs at $t=10 \mathrm{~s}$ (circles: POST2/CFE; lines: AUTOLEV).

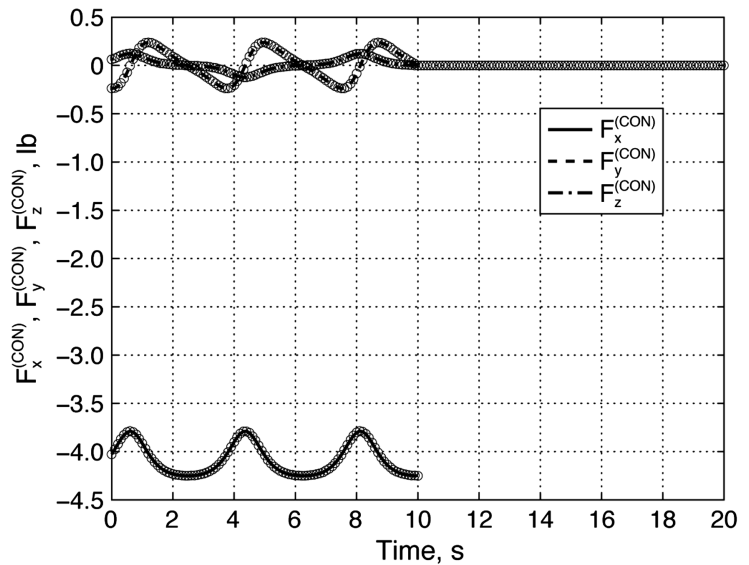

Fig. 11 Test case 1: comparison of constraint forces required to hold bodies together. Joint release occurs at $t=10 \mathrm{~s}$ (circles: POST2/CFE; lines: AUTOLEV).

An important metric to assess the POST2/CFE methodology is the relative joint displacement between the two bodies when they are supposed to stay connected. This parameter is computed as the position vector from $\bar{A}$ to $\bar{B}$ and should be zero while the joint constraint is imposed for the first $10 \mathrm{~s}$. Hence, any deviation from zero is a measure of accuracy of the CFE algorithm. In the CFE algorithm, the constraints are satisfied identically at the acceleration level [Eqs. (12) and (14)]; however, as with algorithms of this type,

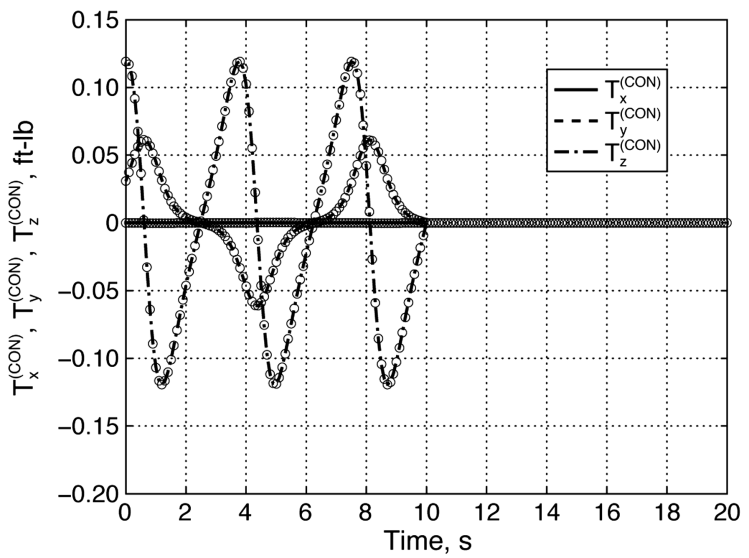

Fig. 12 Test case 1: comparison of constraint torques required to hold bodies together. Joint release occurs at $t=10 \mathrm{~s}$ (circles: POST2/CFE; lines: AUTOLEV).

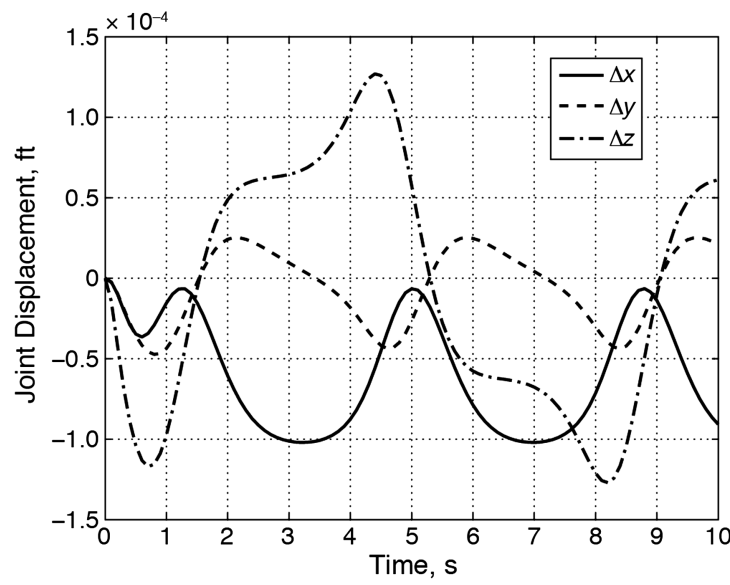

Fig. 13 Test case 1: joint displacement in $x, y$, and $z$ directions for POST2/CFE method during time when bodies are connected. Joint release occurs at $t=10 \mathrm{~s}$.

the constraints at the velocity and position levels are inevitably subject to numerical integration errors. Such errors are functions of the step size in a fixed-step integration scheme or the error limits in a variable-step approach. Baumgarte stabilization controls these errors from growing arbitrarily large during a simulation.

The choice of step size and Baumgarte factor $\eta$ for this problem represents a reasonable balance between $\mathrm{CPU}$ time and error buildup. Figure 13 shows the joint translational displacement as a function of time for the $10 \mathrm{~s}$ period when the bodies are supposed to stay connected. In this paper, the quantities $\Delta x, \Delta y$, and $\Delta z$ are the $x, y$, and $z$ components of joint translational displacement in the local body frame, and they are defined as follows:

$$
\begin{gathered}
\Delta x=\left(\mathbf{r}_{B}-\mathbf{r}_{A}\right) \cdot \hat{\mathbf{a}}_{1}, \quad \Delta y=\left(\mathbf{r}_{B}-\mathbf{r}_{A}\right) \cdot \hat{\mathbf{a}}_{2} \\
\Delta z=\left(\mathbf{r}_{B}-\mathbf{r}_{A}\right) \cdot \hat{\mathbf{a}}_{3}
\end{gathered}
$$

In the interest of clarity, only the POST2/CFE results are shown. The magnitude of this displacement distance is below $0.01 \%$ of the total body length.

\section{B. Test Case 2: Universal Joint}

To check and verify POST2/CFE results, an independent simulation using ADAMS software was generated. At $t=0$, each body was assumed to be at rest. A time-varying force is applied to body $A$ at a location relative to the center of mass given by the vector $-2.0 \hat{\mathbf{a}}_{1}+0.5 \hat{\mathbf{a}}_{3} \mathrm{ft}$. The components of this time-varying force are shown in Fig. 14. The mass properties of each body are summarized in Table 3 . The products of inertia are zero for each body in this problem.

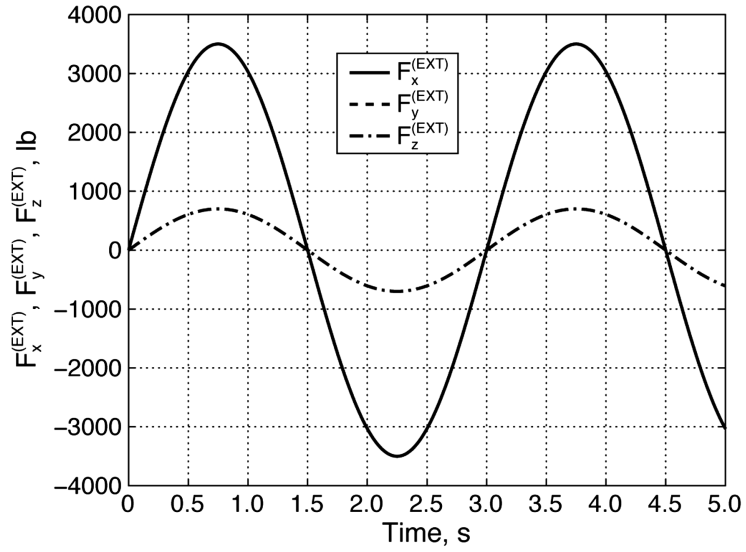

Fig. 14 Test case 2: external force components applied to body A $\left(F_{x}^{(\mathrm{EXT})}=F_{y}^{(\mathrm{EXT})}\right)$. 
The motion of bodies $A$ and $B$ was simulated for a duration of $5 \mathrm{~s}$. The POST2/CFE used a fixed-step-size integrator with a step size of $0.0001 \mathrm{~s}$ and a Baumgarte factor, $\eta=5.0$. The results of the simulations are shown in Figs. 15-18. The angular velocity of body $A$ is shown in Fig. 15. The constraint force and moment components are shown in Figs. 16 and 17, respectively. There is excellent agreement between POST $2 / \mathrm{CFE}$ and the ADAMS solutions, as evidenced by these figures. Finally, the relative joint translational displacement time histories are shown in Fig. 18. As in the previous test case of the

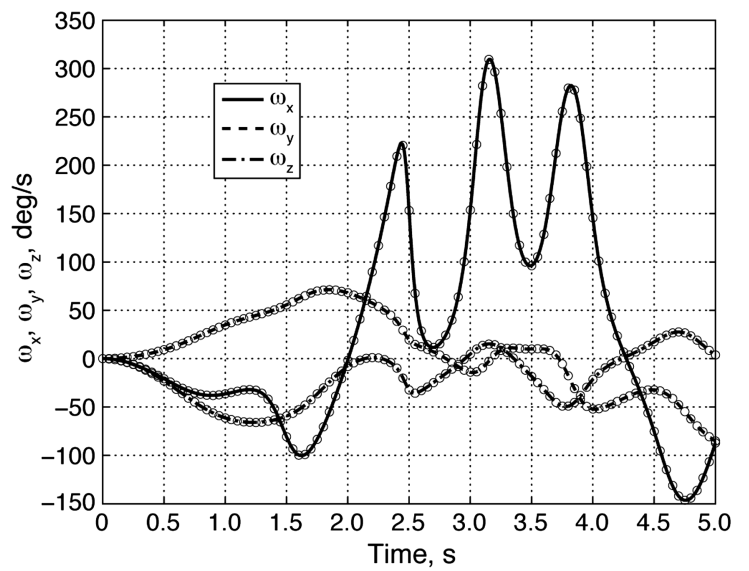

Fig. 15 Test case 2: body $B$ : angular velocity components (circles: POST2/CFE; lines: ADAMS).

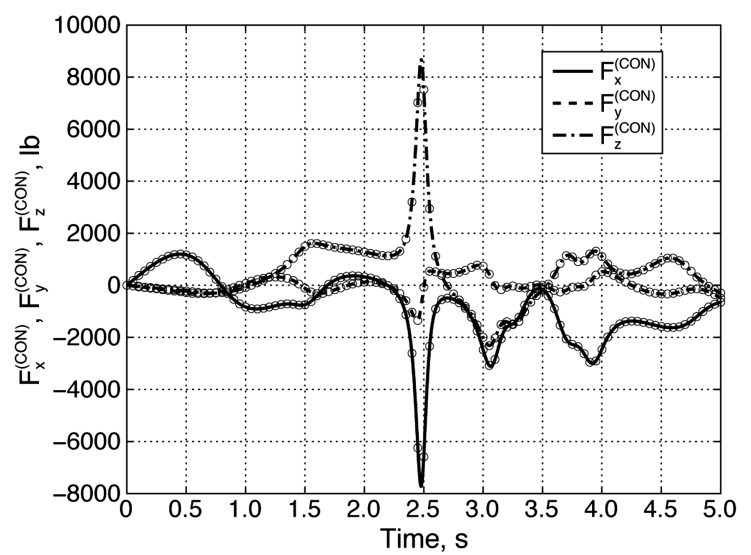

Fig. 16 Test case 2, body $B$ : Comparison of constraint force (circles: POST2/CFE; lines: ADAMS).

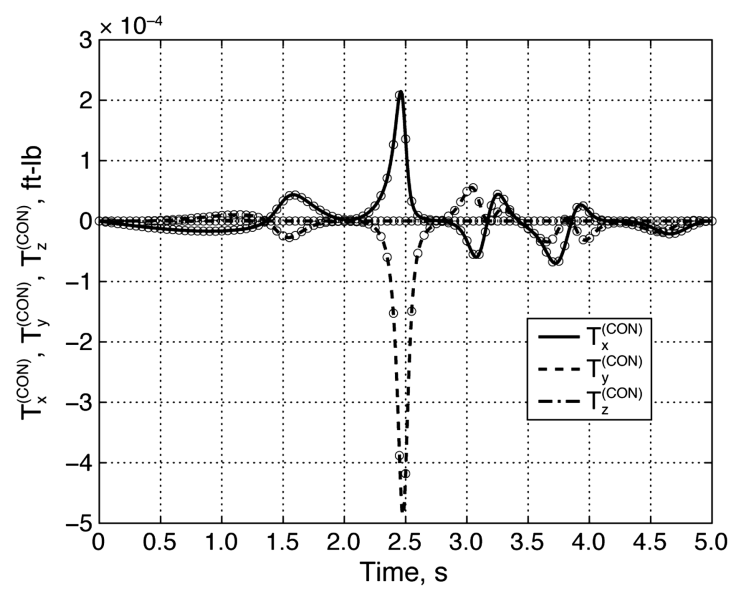

Fig. 17 Test case 2, body $B$ : Comparison of constraint torque (circles: POST2/CFE; lines: ADAMS). fixed joint problem, the joint displacement is relatively small, well below $0.005 \%$ of body length.

\section{Test Case 3: X-43A}

The objective of the third test case was to evaluate the capability of POST2/CFE to model a realistic stage separation problem. For the $\mathrm{X}-43 \mathrm{~A}$ problem that was chosen, POST2/CFE results of the angle of attack and angle of sideslip during separation were compared with data recorded during flight [29]. In addition, because many of the parameters needed to fully verify the POST2/CFE results were not included in the flight data, further comparisons were made with previously published simulation results that were part of the X-43A preflight analysis [30]. This preflight simulation, which was also developed using POST2, modeled each joint between the two pistons and the RV as a massless spring, with a prescribed force profile in the direction along the piston centerline, and with restoring forces computed from beam deflection theory in the lateral directions perpendicular to the axis of piston motion. By modeling the pistons as massless springs, the need to consider constraint equations in the POST2 formulation was avoided; however, the spring-model implementation introduced modeling complexity and required numerous customizations to the POST2 source code.

Reference [30] details the development of this preflight POST2 spring-model simulation and describes the excellent level of agreement between its results and the results of an even higher fidelity simulation of the X-43A stage separation that was developed using ADAMS. Because of this heritage, the POST2 spring-model simulation from [30] was considered suitable for verifying the POST2/CFE simulation results.

A description of how the X-43A stage separation problem was set up in the POST2/CFE formulation was presented in Sec. III. Because of the generalized implementation of the CFE method within POST2, the problem was much easier to set up than the spring-model implementation, and it required few modifications to the POST2 source code. The primary difference in problem setup between the two approaches was in modeling of the pistons, where POST2/CFE modeled only one piston to avoid overconstraining the problem while the spring-model implementation modeled both pistons. Since the objective of the simulation was to determine the motion of both vehicles during separation to assess vehicle recontact potential and optimize control system settings, the details of how the pistons were modeled were not important as long as the motion was constrained the same way while both vehicles were joined. As a measure of how well the joint constraint was satisfied, the relative joint displacement between the two separating vehicles is shown in Fig. 19. This parameter is the distance from the RV joint location to the HXLV joint location, and it was computed in the piston axial (line of action, $\left.\hat{\mathbf{a}}_{1}\right)$, lateral $\left(\hat{\mathbf{a}}_{2}\right)$, and normal $\left(\hat{\mathbf{a}}_{3}\right)$ directions. The results indicate that CFE does a better job of enforcing the translational constraint, and there is virtually no joint displacement in the lateral or normal

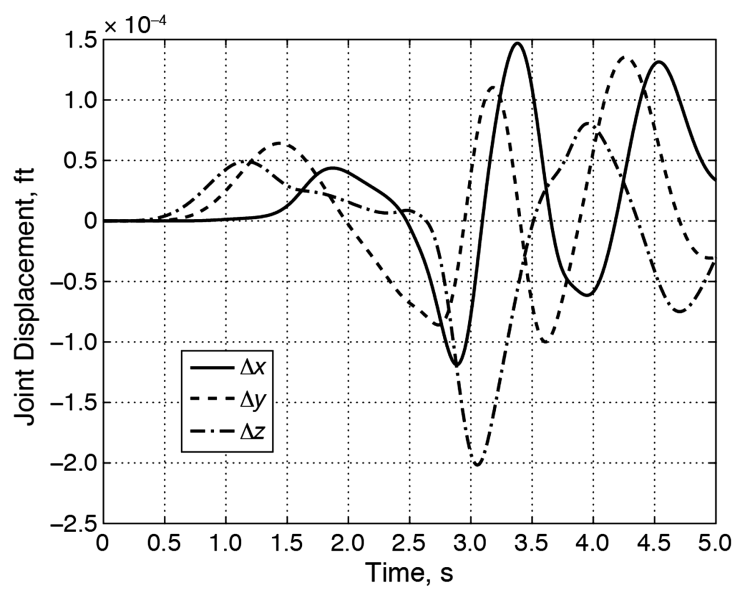

Fig. 18 Test case 2: joint displacement in $x, y$, and $z$ directions for POST2/CFE method during time when bodies are connected. 

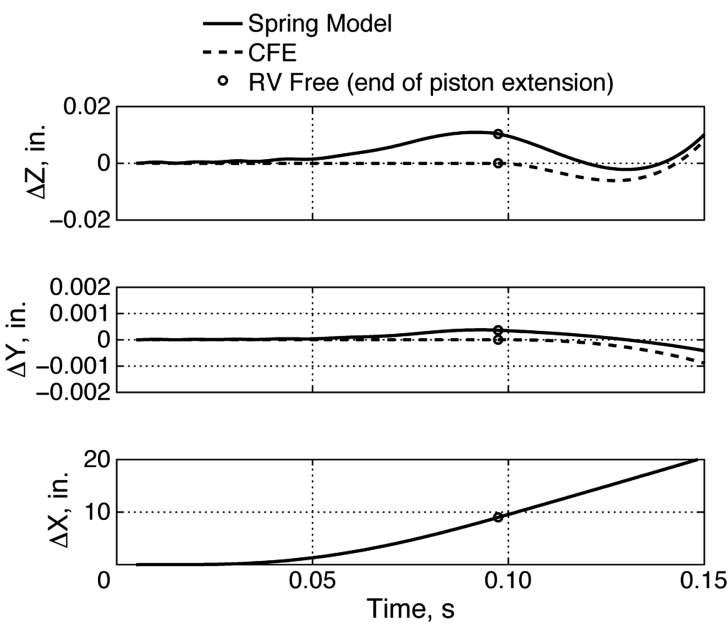

Fig. 19 Test case 3: comparison of joint displacement in $x, y$, and $z$ directions for CFE and spring model.

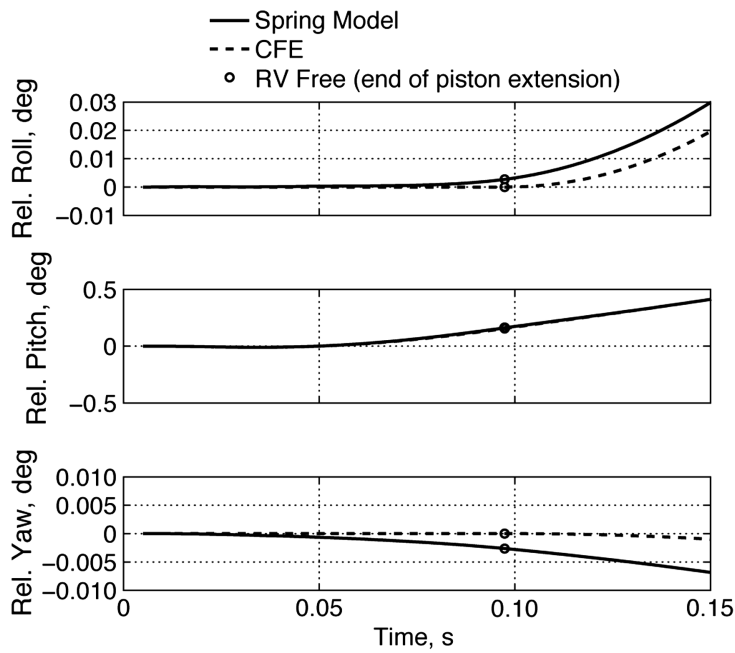

Fig. 20 Test case 3: comparison of relative angular displacement between POST2/CFE and spring model.

directions. Similar results are seen in Fig. 20, which shows the relative difference in attitude between the two vehicles. Again, CFE does an excellent job of enforcing the rotational constraint, essentially eliminating any relative roll and yaw between the RV and HXLV. The constraint forces and moments about the vehicle center of mass that were computed by each technique are shown in Figs. 21 and 22 , respectively. In these plots, the forces and moments
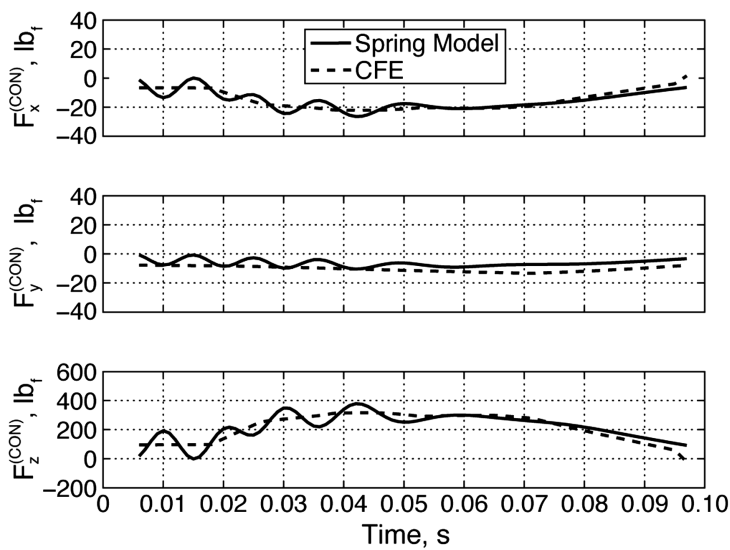

Fig. 21 Test case 3: comparison of constraint forces computed by POST2/CFE and spring model.

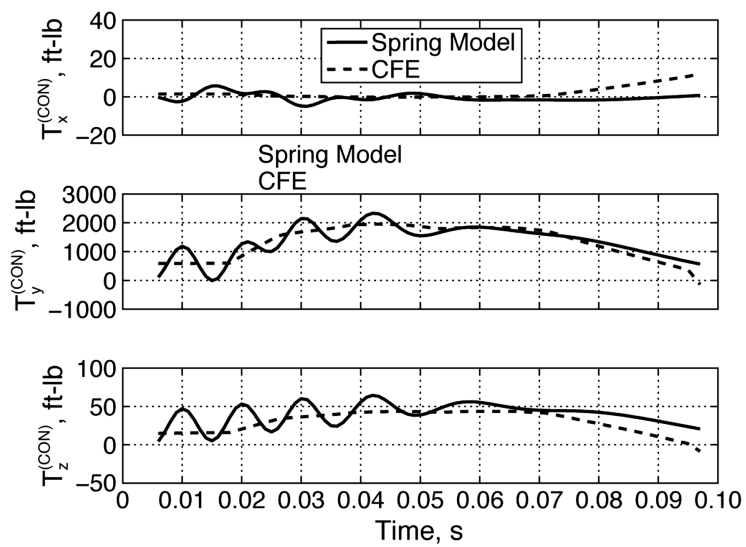

Fig. 22 Test case 3: comparison of constraint moments computed by POST2/CFE and spring model.

computed by the spring model include the contributions of both pistons. The results show that both the spring model and POST2/CFE compute very similar forces and moments. However, the springmodel results are oscillatory in nature, since the joint constraint is not satisfied exactly.

Flight data comparisons of the angle-of-attack and angle-ofsideslip profiles are shown in Fig. 23. Both the spring-model and POST2/CFE results match very well and are nearly identical in the angle-of-attack response. However, there are slightly larger differences in the sideslip response. In addition, both simulation techniques match the observed flight data well. It should be noted that the degree to which the simulation results match the flight data depends on other factors besides CFE modeling, such as aerodynamic force and moments, winds, accuracy of flight data instrumentation, etc. Aerodynamic modeling was especially critical because separation occurred at a dynamic pressure of $1000 \mathrm{psf}$. Thus, this problem is much more complex than the first two test problems where the external forces and moments were prescribed.

To illustrate the sensitivity of the simulation results to aerodynamic modeling, computations were made using the nominal (preflight) aerodynamic database and a postflight reconstructed aerodynamic database in which the uncertainty terms had been adjusted to match the flight data more closely. The variations in angle of attack and sideslip differ significantly depending on which aerodynamic model was used. Note that all POST2/CFE results were generated using the adjusted aerodynamics.

As a further comparison, results are also shown for an unconstrained case where no constraint forces were applied to model the joint (but the piston axial force that pushed the vehicles apart was
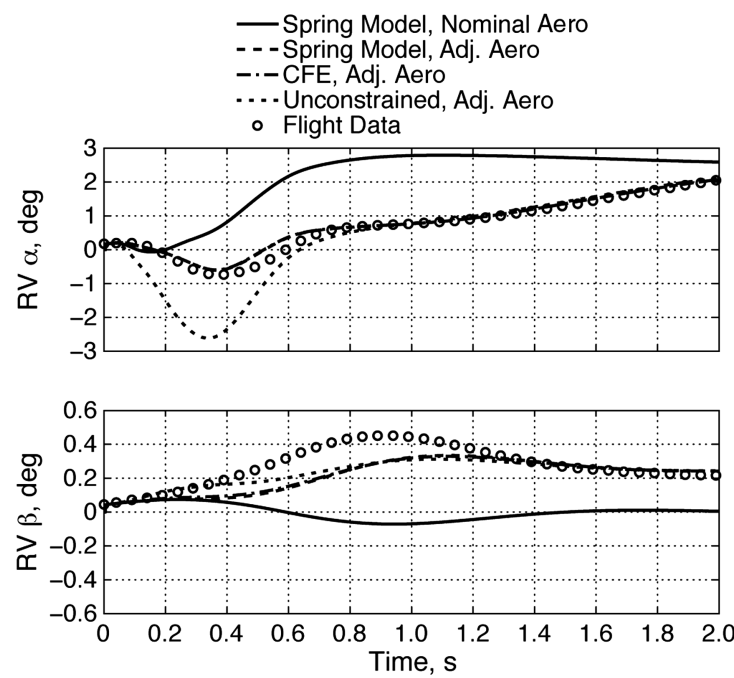

Fig. 23 Test case 3: simulation vs flight data comparison of angle-ofattack and angle-of-sideslip profiles (aero: aerodynamic, adj: adjusted). 

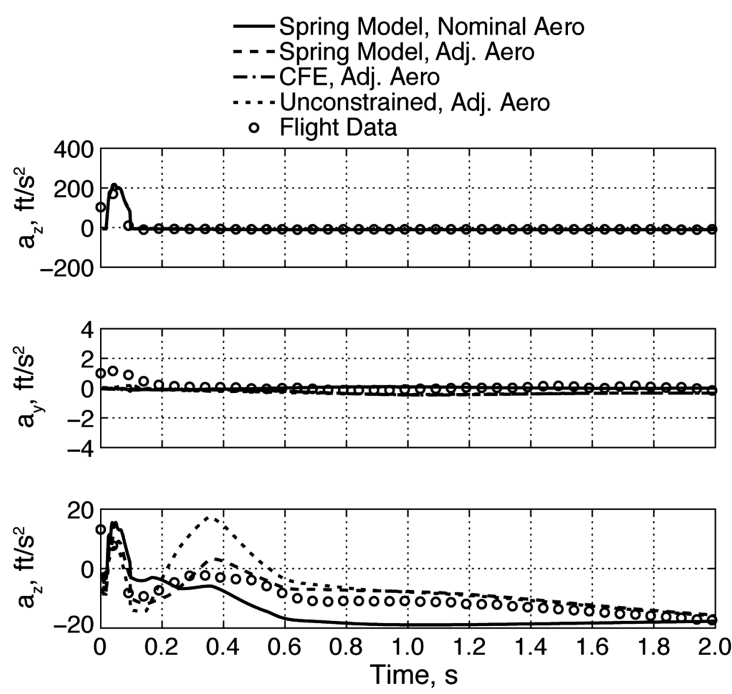

Fig. 24 Test case 3: simulation vs flight data comparison of $x, y$, and $z$ accelerations in local body frame.

still modeled). During the first second of separation, the unconstrained results differed significantly from the cases that included the constraint, demonstrating the importance of accurate modeling of the piston joint. Even though the differences between the constrained and unconstrained angle-of-attack and sideslip profiles disappeared after the control system became active and was able to compensate, it is important to capture the dynamics during the first second of flight, since that was when the risk of recontact was highest.

Similar comparisons are shown for the linear accelerations in Fig. 24. The vertical (i.e., body $z$ direction) acceleration results were the most sensitive to the way in which the piston constraint was modeled, and they were the most important for assessing the risk of recontact. Again, the spring-model and CFE results were nearly identical and provided a good match to the flight data. The differences between the constrained and unconstrained cases were actually larger than the differences due to aerodynamics.

Figure 25 compares the angular velocity results with the flight data. Once again, there is excellent agreement between the springmodel and CFE simulation results. Both simulations match the pitch rate flight data very well and do a much better job at predicting the pitching motion than the unconstrained case. The comparison between simulation and flight for yaw rate and roll rate is not as good. Much of the difference can be attributed to inadequate lateral-
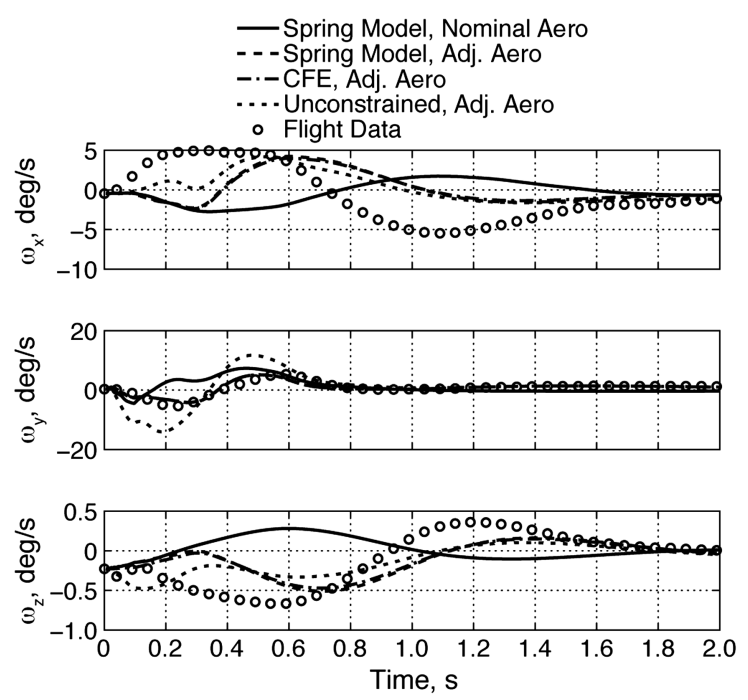

Fig. 25 Test case 3: simulation vs flight data comparison of body roll, pitch, and yaw rate profiles. directional aerodynamic modeling, as is evident in the large difference in results between the cases with nominal aerodynamics and adjusted aerodynamics. Again, capturing the pitch plane dynamics was important for assessing the risk of recontact.

\section{Conclusions}

The CFE methodology provides a simple method to calculate the internal constraint forces and moments acting at joints connecting multiple bodies, and it applies them as external forces and moments. The details of the CFE methodology and its implementation in the POST 2 are discussed in this paper. The CFE methodology is generic in nature and can be implemented in any trajectory simulation software. To illustrate the capability of the CFE algorithm, three test cases of increasing complexity were presented. The first case examined the motion of two force-free rigid bodies connected by a fixed joint, subject to an initial rotation. The simple nature of this problem made it possible to compare simulation results to analytical predictions based on first principles. In addition, simulating a fixed joint presented a challenging case with which to assess the relative displacement between the two bodies at the joint location, a key performance metric for the CFE algorithm. The second case involved rigid bodies connected by a universal joint, and it typified the testing that was performed on a number of different joints applicable to stage separation problems. The CFE results were in excellent agreement with results obtained using AUTOLEV in the first test case and with those obtained using ADAMS in the second case.

In the third test case, the ability of the CFE method to model realistic launch vehicle stage separation was demonstrated by simulating X43-A stage separation. These results agreed well with previously published results that were based on an engineering model, and they actually performed better at satisfying the joint constraint. Moreover, the CFE results agreed reasonably well with the flight data, and it was shown that the level of agreement degraded significantly if the constraint was not modeled.

The success of these test cases provides confidence in the ability of the CFE methodology to solve constrained motion problems in conventional trajectory simulation programs and to provide the capability of performing generic end-to-end simulations of launch vehicle trajectories, including stage separation.

\section{Acknowledgments}

The authors gratefully acknowledge Anne Rhodes for her invaluable assistance in preparing this paper for publication and Jim Beaty for his helpful comments.

\section{References}

[1] Dillenius, M. F. E., Perkins, S. C., and Nixon, D., "Pylon Carriage and Separation of Stores," Tactical Missile Aerodynamics-General Topics, edited by M. J. Hemsch, Progress in Astronautics and Aeronautics, AIAA, Washington, D.C., 1992, pp. 575-666.

[2] Taylor, R. T., and Alford, W. J., Jr., "A Wind Tunnel Investigation of the Carry Loads and Mutual Interference Effects of 1/40-Scale Models of the X-15 and B-52 Airplanes In Combination," NASA TM X-184, 1959.

[3] Decker, J. P., and Wilhite, A. W., "Technology and Methodology of Separating Two Similar Size Aerospace Vehicles Within the Atmosphere," AIAA Paper 1975-0029, Jan. 1975.

[4] Decker, J. P., "Experimental Aerodynamics and Analysis of the Stage Separation of Reusable Launch Vehicles," NASA, SP-148, 1967.

[5] Decker, J. P., and Gera, J., "An Exploratory Study of Parallel-Stage Separation of Reusable Launch Vehicles," NASA TN D-4765, 1968.

[6] Decker, J. P., "Aerodynamic Interference Effects Caused by ParallelStaged Simple Aerodynamic Configuration at Mach Numbers of 3 and 6," NASA TN D-5379, 1969.

[7] Wilhite, A. W., "Analysis of Separation of the Space Shuttle Orbiter from a Large Transport Airplane," NASA TM X-3492, 1977.

[8] Naftel, J. C., and Wilhite, A. W., "Analysis of Separation of a Two-Stage Winged Launch Vehicle," AIAA Paper 1986-0195, Jan. 1986.

[9] Naftel, J. C., and Powell, R. W., "Aerodynamic Separation and Glideback of a Mach 3 Staged Orbiter," AIAA Paper 1990-0223, Jan. 1990. 
[10] Naftel, J. C., and Powell, R. W., "Analysis of the Staging Maneuver and Booster Glideback Guidance for a Two-Staged, Winged, Fully Reusable Launch Vehicle," NASA TP-3335, 1993.

[11] Murphy, K. J., Buning, P. G., Pamadi, B. N., Scallion, W. I., and Jones, K. M., "Status of Stage Separation Tool Development for Next Generation Launch Vehicle Technologies," AIAA Paper 2004-2595, 2004.

[12] Using ADAMS/Solver, Mechanical Dynamics, Ann Arbor, MI, 1999.

[13] Pamadi, B. N., Neirynck, T. A., Covell, P. F., Hotchko, N. J., and Bose, D. M., "Simulation and Analyses of Staging Maneuvers of Next Generation Reusable Launch Vehicles," AIAA Paper 2004-5185, 2004.

[14] Pamadi, B. N., Neirynck, T. A., Hotchko, N. J., Scallion, W. I., Murphy, K. J., and Covell, P. F., "Simulation and Analyses of Stage Separation of Two-Stage Reusable Launch Vehicles," Journal of Spacecraft and Rockets, Vol. 44, No. 1, 2007, pp. 66-80. doi: $10.2514 / 1.17896$

[15] Pamadi, B. N., Hotchko, N. J., Samareh, J., Covell, P. F., and Tartabini, P. V., "Simulation and Analyses of Multi-Body Separation in Launch Vehicle Staging Environment," AIAA Paper 2006-8033, 2006

[16] Toniolo, M. D., Tartabini, P. V., Pamadi, B. N., and Hotchko, N. J., "Constraint Force Equation Methodology for Modeling Multi-Body Stage Separation Dynamics," AIAA Paper 2008-0219, 2008.

[17] Brauer, G. L., Cornick, D. E., and Stevenson, R., "Capabilities and Applications of the Program to Optimize Simulated Trajectories (POST)," NASA CR-2770, 1977.

[18] Striepe, S. A., Powell, R. W., Desai, P. N., Queen, E. M., Brauer, G. L., Cornick, D. E., Olson, D. W., Petersen, F. M., Stevenson, R., Engel, M. C., Marsh, S. M., and Gromko, A. M., Program to Optimize Simulated Trajectories (POST II), Vol. 2: Utilization Manual, Ver. 1.1.7, NASA Langley Research Center, Hampton, VA, July 2002.

[19] Baumgarte, J., "Stabilization of Constraints and Integrals of Motion in Dynamical Systems," Computer Methods in Applied Mechanics and Engineering, Vol. 1, No. 1, 1972, pp. 1-16. doi:10.1016/0045-7825(72)90018-7

[20] Greenwood, D. T., Principles of Dynamics, Prentice-Hall, Englewood
Cliffs, NJ, 1965, pp. 267-269.

[21] Wang, J. T., and Huston, R. L., "Kane's Equations with Undetermined Multipliers: Application to Constrained Multibody Systems," Journal of Applied Mechanics, Vol. 54, No. 2, 1987, pp. 424-429. doi:10.1115/1.3173031

[22] Anderson, K. S., "An Order $n$ Formulation for the Motion Simulation of General Multi-Rigid-Body Constrained Systems," Computers and Structures, Vol. 43, No. 3, 1992, pp. 565-579. doi:10.1016/0045-7949(92)90289-C

[23] Toniolo, M. D., "POST2 CFE Updates and Verification Final Report," Analytical Mechanics Assoc., AMA Rept. 10-04, Hampton, VA, Jan. 2010

[24] McClinton, C. R., "X-43 Scramjet Power Breaks the Hypersonic Barrier, Dryden Lectureship in Research for 2006," AIAA Paper 2006$0001,2006$.

[25] Schaechter, D. B., and Levinson, D. A., "Interactive Computerized Symbolic Dynamics for the Dynamicist," Journal of the Astronautical Sciences, Vol. 36, No. 4, Oct.-Dec. 1988, pp. 365-388.

[26] Kane, T. R., and Levinson, D. A., AUTOLEV 4 User's Manual, OnLine Dynamics, Sunnyvale, CA, 2005

[27] Kane, T. R., and Levinson, D. A., Dynamics Online: Theory and Implementation With AUTOLEV, OnLine Dynamics, Sunnyvale, CA, 2000.

[28] Kane, T. R., and Levinson, D. A., Dynamics: Theory and Applications, McGraw-Hill, New York, 1985, pp. 158-166.

[29] Karlgaard, C. D., Tartabini, P. V., Blanchard, R. C., Kirsch, M., and Toniolo, M. D., "Hyper-X Post-Flight Trajectory Reconstruction," Journal of Spacecraft and Rockets, Vol. 43, No. 1, 2006, pp. 105-115. doi: $10.2514 / 1.12733$

[30] Tartabini, P. V., Bose, D. M., McMinn, J. D., Martin, J. G., and Strovers, B. K., "Hyper-X Stage Separation Trajectory Validation Studies," AIAA Paper 2003-5819, Aug. 2003.

M. Miller

Associate Editor 